\title{
Casimir-Polder Interaction in the Presence of Parallel Walls
}

\author{
F. C. Santos, J. J. Passos Sobrinho, and A. C. Tort \\ Instituto de Física, Universidade Federal do Rio de Janeiro \\ Cidade Universitária, Ilha do Fundão, CP 68528, 21945-970 Rio de Janeiro RJ, Brazil
}

Received on 9 March, 2004; revised version received on 2 October, 2004

\begin{abstract}
Making use of the quantum correlators associated with the Maxwell field vacuum distorted by the presence of plane parallel material surfaces we rederive the Casimir-Polder interaction in the presence of plane parallel conducting walls. For a configuration consisting of a conducting wall and a magnetically permeable one new results for the Casimir-Polder interaction potential are obtained.
\end{abstract}

In 1948, Casimir and Polder [1] taking into account a suggestion made by experimentalists evaluated the interaction potential between two eletrical polarizable molecules separated by a distance $r$ including the effects due to the finiteness of the speed of propagation of the electromagnetic interaction, i.e.: of the retardment. Casimir and Polder showed that the retardment causes the interaction potential to change from a $r^{-6}$ power law to a $r^{-7}$ power law. In the same paper, Casimir and Polder also analyzed the retarded interaction between an atom and a conducting wall and showed that the interaction potential in this case varies according to a $r^{-4}$ power law, where now $r$ is the distance between the atom and the wall. For an introduction to these subjects see [2]. Here we wish to show how it is possible with the help of the so called renormalized electromagnetic field correlators, in our case the ones that take into account the presence of the boundary conditions imposed on the fields, to reobtain the piece of Casimir and Polder's result for the atom-wall interaction that depends on the distortion of the vacuum oscillations of the electromagnetic field caused by the presence of parallel walls. The electromagnetic field correlators for the case of two parallel perfectly conducting surfaces separated by a distance $a$ were evaluated in [3] and in [4]. For the case of a perfectly conducting plane wall and a perfectly permeable plane wall, a setup first introduced by Boyer [5], they were calculated in [4]. These mathematical objects, closely related to the pertinent electromagnetic Green's functions, were also employed to obtain an alternative view of the Casimir effect [6] through the quantum version of the Lorentz force between the walls [7].

Let us first recall some aspects concerning electrically and magnetically polarizable bodies [8]. From a classical point of view the induced eletrical polarization density $\mathbf{P}$ can be thought of as a function of the electric and magnetic fields $\mathbf{E}$ and $\mathbf{B}$. In many cases only the dependence on the eletric field is relevant. It can be shown that under conditions for which the effects of the retardment (i.e., of the finiteness of the speed of light) must be taken into account it suffices to consider the static eletrical polarizability $\alpha(0)$ only, see for instance [2] and references therein. If the electric field changes by $\delta \mathbf{E}$, the interaction between the polarizable body and the electric field will change according to $\delta V=-\mathbf{P}[\mathbf{E}] \cdot \delta \mathbf{E}=-\alpha(\mathbf{0}) \mathbf{E} \cdot \delta \mathbf{E}$. Therefore, if the field changes from zero to a finite value $\mathbf{E}$, the interaction energy is $V_{E}=-\alpha(0) \mathbf{E}^{2} / \mathbf{2}$. In the quantum version of this interaction potential we must replace $\mathbf{E}^{2}$ by its vacuum expectation value, $\left\langle\hat{\mathbf{E}}^{2}\right\rangle_{0}$. The same arguments hold when we consider the magnetization $\mathbf{M}$. The quantum interaction potential between a magnetically polarizable atom and the magnetic field is given by $V_{M}=-\beta(0)\left\langle\mathbf{B}^{\mathbf{2}} / \mathbf{2}\right\rangle_{0}$, where $\beta(0)$ is the static magnetic polarizability. In order to proceed we must know the vacuum expectation values of the quantum field operators $\mathbf{E}^{\mathbf{2}}$ and $\mathbf{B}^{\mathbf{2}}$. This means to evaluate explicitly the vacuum expectation values of the so called electromagnetic field correlators $E_{i}(\mathbf{r}, \mathbf{t}) E_{j}(\mathbf{r}, \mathbf{t})$, $B_{i}(\mathbf{r}, \mathbf{t}) B_{j}(\mathbf{r}, \mathbf{t})$, and $E_{i}(\mathbf{r}, \mathbf{t}) B_{j}(\mathbf{r}, \mathbf{t})$, in the presence of external conditions, i.e., boundary conditions. A regularization recipe will also be necessary. Fortunately these objects were calculated before and we can limit ourselves to make use of the results.

For the case of two parallel conducting walls separated by a fixed distance $a$ we have $[3,4]$

$$
\begin{aligned}
& \left\langle E_{i}(\mathbf{r}, \mathbf{t}) \mathbf{E}_{\mathbf{j}}(\mathbf{r}, \mathbf{t})\right\rangle_{\mathbf{0}}= \\
& =\left(\frac{\pi}{a}\right)^{4} \frac{2}{3 \pi}\left[\left(-\delta^{\|}+\delta^{\perp}\right)_{i j} \frac{1}{120}+\delta_{i j} F(\xi)\right],
\end{aligned}
$$

where $\delta_{i j}^{\|}:=\delta_{i x} \delta_{j x}+\delta_{i y} \delta_{j y}$ and $\delta_{i j}^{\perp}:=\delta_{i z} \delta_{j z}$. The function $F(\xi)$ with $\xi:=\pi z / a$ is defined by

$$
F(\xi):=-\frac{1}{8} \frac{d^{3}}{d \xi^{3}} \frac{1}{2} \cot (\xi),
$$

and its expansion about $\xi=0$ is given by

$$
F(\xi) \approx \frac{3}{8} \xi^{-4}+\frac{1}{120}+O\left(\xi^{2}\right)
$$

Near $\xi=\pi$ (which corresponds to $z=a$ ) we make the replacement $\xi \rightarrow \xi-\pi$. Notice that due to the behavior of 
$F(\xi)$ near $\xi=0, \pi$, divergences control the behavior of the correlators near the plates.

The magnetic field correlators are $[3,4]$

$$
\begin{aligned}
& \left\langle B_{i}(\mathbf{r}, \mathbf{t}) \mathbf{B}_{\mathbf{j}}(\mathbf{r}, \mathbf{t})\right\rangle_{\mathbf{0}}= \\
& =\left(\frac{\pi}{a}\right)^{4} \frac{2}{3 \pi}\left[\left(-\delta^{\|}+\delta^{\perp}\right)_{i j} \frac{1}{120}-\delta_{i j} F(\xi)\right] .
\end{aligned}
$$

A direct evaluation shows that the correlators < $\left.E_{i}(\mathbf{r}, \mathbf{t}) \mathbf{B}_{\mathbf{j}}(\mathbf{r}, \mathbf{t})\right\rangle_{\mathbf{0}}$ are zero.

For the case of a perfectly conducting plane wall and a magnetically permeable one results are [4]

$$
\begin{aligned}
& \left\langle\hat{E}_{i}(\mathbf{r}, \mathbf{t}) \hat{E}_{j}(\mathbf{r}, \mathbf{t})\right\rangle_{0}= \\
& =\left(\frac{\pi}{a}\right)^{4} \frac{2}{3 \pi}\left[\left(-\frac{7}{8}\right) \frac{\left(-\delta^{\|}+\delta^{\perp}\right)_{i j}}{120}+\delta_{i j} G(\xi)\right],
\end{aligned}
$$

and

$$
\begin{aligned}
& \left\langle\hat{B}_{i}(\mathbf{r}, \mathbf{t}) \hat{B}_{j}(\mathbf{r}, \mathbf{t})\right\rangle_{0}= \\
& =\left(\frac{\pi}{a}\right)^{4} \frac{2}{3 \pi}\left[\left(-\frac{7}{8}\right) \frac{\left(-\delta^{\|}+\delta^{\perp}\right)_{i j}}{120}-\delta_{i j} G(\xi)\right],
\end{aligned}
$$

where the function $G(\xi)$ is defined by

$$
G(\xi)=-\frac{1}{8} \frac{d^{3}}{d \xi^{3}} \frac{1}{2 \sin (\xi)} .
$$

Observe that near $\xi=0$ the function $G(\xi)$ behaves as

$$
G(\xi)=\frac{3}{8} \xi^{-4}-\frac{7}{8} \frac{1}{120}+O\left(\xi^{2}\right),
$$

near $\xi=\pi$, however, its behavior is slightly different

$$
G(\xi)=-\frac{3}{8}(\xi-\pi)^{-4}+\frac{7}{8} \frac{1}{120}+O\left[(\xi-\pi)^{2}\right] .
$$

Again, a direct calculation shows that $\left\langle\hat{E}_{i}(\mathbf{r}, \mathbf{t}) \hat{B}_{j}(\mathbf{r}, \mathbf{t})\right\rangle_{0}=$ 0 for this case also. As before the divergent behavior of the correlators near the plates we are interested in is an effect of the distortions of the electromagnetic oscillations with respect to a situation where the plates are not present. The correlators given by (2), (5), (6), and (7) allow us to obtain in a straightforward way expressions for the interaction potential energy between an electrically or magnetically polarizable atom placed between the walls and the walls.

Let us consider first the case of an electrically polarizable atom or molecule placed between two perfectly conducting parallel walls. Suppose that the atom is placed at a distance $z$ from the conducting wall placed at $z=0$. The interaction potential between the atom and the walls is given by

$$
V_{E}(z)=-\frac{1}{2} \alpha(0)\left\langle\hat{\mathbf{E}}^{2}(\mathbf{z})\right\rangle_{0}
$$

where $\alpha(0)$ is the static polarizability of the molecule. Making use of (2) we can evaluate $\left\langle\hat{\mathbf{E}}^{2}(\mathbf{z})\right\rangle_{0}$ and using the above equation we obtain

$$
V_{E}(z)=-\frac{\alpha(0) \pi^{3}}{3 a^{4}}\left[3 F\left(\frac{\pi z}{a}\right)-\frac{1}{120}\right] .
$$

Making use of (3) and taking the limit $a \rightarrow \infty$ we obtain the single wall limit of the interaction potential between an electrically polarizable atom and a conducting wall,

$$
V_{E}(z)=-\frac{3 \alpha(0)}{8 \pi z^{4}}
$$

in agreement with [9, 10]; see also [2]. Consider now a magnetically polarizable atom or molecule placed between the two conducting walls. The interaction potential in this case will be given by

$$
V_{M}(z)=+\frac{\beta(0) \pi^{3}}{3 a^{4}}\left[3 F\left(\frac{\pi z}{a}\right)+\frac{1}{120}\right],
$$

where we made use of (5). If the atom or molecule is simultaneously electrically and magnetically polarizable the interaction potential will be simply $V(z)=V_{E}(z)+V_{M}(z)$, that is

$$
\begin{aligned}
V(z) & =-(\alpha(0)-\beta(0)) \frac{\pi^{3}}{a^{4}} F\left(\frac{\pi z}{a}\right) \\
& +(\alpha(0)+\beta(0)) \frac{\pi^{3}}{360 a^{4}} .
\end{aligned}
$$

The single conducting wall limit $(a \rightarrow \infty)$ of (14) is easily obtained with the help of (3). The result is:

$$
V(z) \approx-\frac{3}{8 \pi z^{4}}(\alpha(0)-\beta(0))
$$

which is in agreement with $[9,10]$.

The polarizable atom or molecule can be also placed between a conducting plate at $z=0$ and a permeable one at $z=a$. In this case, making use of (6) e (7) a straightforward calculation leads to the following result

$$
\begin{gathered}
V(z)=-(\alpha(0)-\beta(0)) \frac{\pi^{3}}{a^{4}} G\left(\frac{\pi z}{a}\right) \\
+(\alpha(0)+\beta(0))\left(-\frac{7}{8}\right) \frac{\pi^{3}}{360 a^{4}} .
\end{gathered}
$$

There are now two single walls limits to be considered. Near the conducting plate at $z=0$ the potential is given by (15), but near the perfectly permeable plate at $z=a$, the potential is repulsive and given by

$$
V(z) \approx+\frac{3}{8 \pi(z-a)^{4}}(\alpha(0)-\beta(0)),
$$

where we made use of (9). Fig. 1 shows the behavior of a slightly modified version of Eq. (16) as a function of the distance between the plates. Notice that the interaction potential diverges sharply near the plates. These last results are to our knowledge new. 


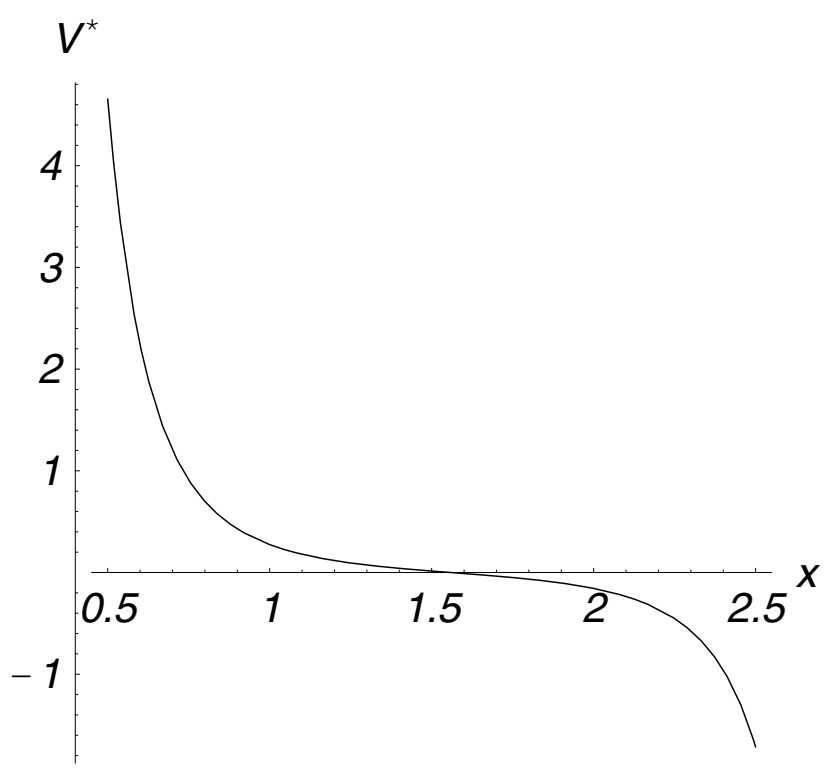

Figure 1. The plot shows the potential $V^{*}:=a^{4} V / \pi^{3} \beta(0)$ obtained from Eq. (16) for $\beta=(9 / 2) \alpha$, which holds for the hydrogen atom at low temperatures. The vertical axis was conveniently shifted toward the right. The horizontal axis is $x:=\pi z / a$.

Nothing new is obtained if we consider a setup formed by two infinitely permeable plates.

It is important to keep in mind that, as mentioned before, we have dealt with a part of the interaction between an atom and two or one walls. The contribution of the interaction between the electric/magnetic dipole moment and its images was utterly neglected. Therefore, the results refer only to the contribution of the quantum vacuum distorted by one or two walls to the total interaction potential. With this proviso we can state that the Casimir-Polder interaction shows certain aspects of the quantum structure of the vacuum confined between the plane surfaces in question. The atom acts as a probe of the confined quantum vacuum, particularly near the walls.

\section{References}

[1] H. B. G. Casimir and D. Polder, Phys. Rev 73, 360 (1948).

[2] P.W. Milonni, The Quantum Vaccum: An introduction to Quantum Electrodynamics, (Academic Press, New York, 1994).

[3] C. A. Lütken and F. Ravndal, Phys. Rev. A 31, 2082 (1985); see also: G. Barton, Phys. Lett. B 237, 559 (1990); M. Bordag, D. Robaschik and E. Wieczorek, Ann. Phys. (NY) 165, 192 (1985).

[4] M. V. Cougo-Pinto, C. Farina, F. C. Santos, and A. C. Tort, J. Phys. A: Math. Gen. 32, 4463 (1999).

[5] T. H. Boyer Phys. Rev A 9, 2078 (1974).

[6] H. B. G. Casimir, Proc. K. Ned. Akad. Wet. 51, 793 (1948); Philips Res. Rep. 6162 (1951).

[7] C. Farina, F. C. Santos, and A. C. Tort, Eur. J. Phys. 24, N1N5 (2003).

[8] J. D. Jackson, Classical Electrodynamics, 3rd. ed., (John Wiley, New York 1999).

[9] H. B. G. Casimir, J. Chim. Phys. 46, 407 (1948).

[10] T. H. Boyer, Phys. Rev. 180, 19 (1969). 